\title{
CEACAM6 promotes cholangiocarcinoma migration and invasion by inducing epithelial-mesenchymal transition through inhibition of the SRC/PI3K/AKT signaling pathway
}

\author{
CHEN LIU ${ }^{1}$, MIN WANG $^{2}$, HAITAO LV ${ }^{1}$, BING LIU ${ }^{3}$, XUEQIANG YA ${ }^{1}$, \\ WEIHONG ZHAO $^{1}$ and WENBIN WANG ${ }^{1}$ \\ Departments of ${ }^{1}$ Hepatobiliary Surgery and ${ }^{2}$ Respiratory and Critical Care Medicine, \\ The Second Hospital of Hebei Medical University; ${ }^{3}$ Department of Oncology, \\ The Third Hospital of Hebei Medical University, Shijiazhuang, Hebei 050000, P.R. China
}

Received October 16, 2020; Accepted October 15, 2021

DOI: $10.3892 / \mathrm{ol} .2021 .13157$

\begin{abstract}
The immunoglobulin superfamily member carcinoembryonic antigen-related cell adhesion molecule 6 (CEACAM6) is overexpressed in a wide variety of human cancer types, and is associated with tumor invasion and migration. The aim of the present study was to determine the role of CEACAM6 in cholangiocarcinoma (CCA) invasion and migration in vitro. The results showed that CEACAM6 was highly expressed in CCA tissues, and that the expression level of CEACAM6 was negatively associated with the degree of differentiation of CCA. Silencing CEACAM6 inhibited cell viability, invasion and migration but promoted cell apoptosis in a human CCA cell line (RBE). In addition, CEACAM6 knockdown decreased the expression of an antiapoptotic protein (Bcl-2), an interstitial cell marker (N-cadherin), extracellular matrix proteins (MMP-2 and MMP-9), a transcription factor helix protein (Twist-related protein 1), an intermediate tumor cell scaffold marker (vimentin), a protein involved in tumor nutrient vascular formation (VEGFA) and a tumorigenesis factor (intercellular cell adhesion molecule-1), but increased the expression of pro-apoptotic proteins (Bax, and cleaved caspases-3, -8 and -9) and an epithelial cell marker protein (E-cadherin). Furthermore, CEACAM6-small interfering RNA reduced the expression of the SRC/PI3K/AKT signaling transduction pathway. Taken together, these results suggested that CEACAM6 may be an epithelial-mesenchymal transition biomarker and a potential therapeutic target in human CCA.
\end{abstract}

Correspondence to: Dr Wenbin Wang, Department of Hepatobiliary Surgery, The Second Hospital of Hebei Medical University, 215 Heping West Road, Shijiazhuang, Hebei 050000, P.R. China E-mail: wwb2981@163.com

Key words: carcinoembryonic antigen-related cell adhesion molecule 6, cholangiocarcinoma, migration, invasion, epithelialmesenchymal transition

\section{Introduction}

Cholangiocarcinoma (CCA) has been reported to be the second most common hepatic malignancy after hepatocellular carcinoma (HCC), and its incidence has gradually increased worldwide (1-2). CCA is a relatively infrequent malignancy arising from epithelial cells lining the biliary tree, and is classified anatomically as intrahepatic (iCCA), perihilar (pCCA) or distal (3). The incidence of iCCA has increased globally over the past few decades. According to the mortality rates reported in the World Health Organization database, the age-standardized mortality rates for iCCA have increased in almost all countries across all continents (4). Surgery is the preferred treatment option for a minority of patients with early-stage CCA ( 35\%). However, the currently available systemic therapies are of limited effectiveness for patients with advanced-stage or unresectable CCA, due to its absence of clinical symptoms and anatomical location of difficult access (5). Therefore, it is urgent to explore novel markers and molecules for CCA therapy.

Epithelial-mesenchymal transition (EMT) is associated with tumor invasion and migration, and is defined as a reversible dynamic process in which epithelial cells lose their phenotypic characteristics and adopt mesenchymal cell structural and functional features $(6,7)$. These changes involve downregulation of E-cadherin and $\beta$-catenin, which are two main phenotypic characteristic components of epithelial cells, as well as upregulation of proteins involved in the mesenchymal phenotype ( $\mathrm{N}$-cadherin, $\alpha$-smooth muscle actin, vimentin, fibronectin and MMPs), which occur via EMT-inducing transcription factors. The EMT-inducing transcription factor comprise three families: Snail, zinc finger E-box-binding homeobox and Twist-related proteins (TWIST) (8).

EMT is involved in human physiological and pathological processes, including embryonic and organ development, organ fibrosis, wound healing, and cancer progression $(9,10)$, and is accompanied by marked changes in cellular morphology, enhanced migratory and invasive capabilities, as well as loss and remodeling of cell-cell interactions and cell-matrix adhesion (11). Thus, EMT may be an important mechanism involved 
in the progression of CCA, and EMT biomarkers may provide new insights into the progression and migration of CCA. In addition, the activities of cellular (c)-SRC kinase, and PI3K and AKT, which are potentially located downstream of c-SRC, are hallmarks for malignant transformation and progression.

Carcinoembryonic antigen-related cell adhesion molecule 6 (CEACAM6), a member of the CEACAM family, is normally expressed on the surface of myeloid and epithelial cells, while aberrant CEACAM6 expression leads to the development of human malignancies (12-14). Previous studies have reported that CEACAM6 is overexpressed in several epithelial carcinomas, including colon, breast and non-small cell lung cancer, as well as iCCA $(15,16)$. CEACAM6 mediates cell-cell adhesion, which is required for guiding cells to their correct location during embryonic development, and for the integration of single cells into functional tissues and organs (17). However, overexpression of CEACAM6 has been shown to alter tissue architecture in several carcinoma cell lines (18). In addition, overexpression of CEACAM6 reduces cell apoptosis, indicating that CEACAM6 plays a key role in promoting the aberrant growth of adherent cells (19). Previous studies have shown that CEACAM6 induces EMT, and mediates invasion and migration in pancreatic and gastric cancer, and its clinical importance in colorectal cancer has also been reported $(20,21)$. However, the physiological function of CEACAM6, and its potential involvement in tumor formation and progression in CCA have not been fully elucidated to date.

The present study demonstrated that CEACAM6 is an important regulator of CCA EMT, as well as of cell migration and invasion in vitro, which suggests that CEACAM6 may be an important target for the treatment of human CCA.

\section{Materials and methods}

Tissues and cell line. Primary CCA tissues and matched adjacent paracancerous tissues (distance, $1 \mathrm{~cm}$ ) were surgically obtained from 27 patients with CCA 20 males and 7 females with an age range of 56-78 years (mean age, 66.4 years), including 9 cases of highly differentiated CCA and 18 cases of less differentiated CCA) at The Second Hospital of Hebei Medical University (Shijiazhuang, China) from January 2016 to December 2017. Ethics approval (approval no. 2014018) was obtained from The Second Hospital of Hebei Medical University and all samples were collected with written informed consent from the patients.

RBE cells (Qiao Xinzhou Biotechnology Co., Ltd.) were cultured at $37^{\circ} \mathrm{C}$ with $5 \% \mathrm{CO}_{2}$ in DMEM (Thermo Fisher Scientific, Inc.) containing $10 \%$ fetal bovine serum (FBS; Biological Industries; Sartorius AG) and $1 \%$ penicillin and streptomycin (Sigma-Aldrich; Merck KGaA). Cells in the logarithmic growth phase were used for experiments.

Reverse transcription-quantitative PCR (RT-qPCR). Total RNA was extracted from CCA and paracancerous tissue and RBE cells using TRIzol ${ }^{\circledR}$ reagent (Invitrogen; Thermo Fisher Scientific, Inc.), and first-strand cDNA was synthesized using PrimeScript $^{\mathrm{TM}}$ RT Reagent kit (Takara Biotechnology Co., Ltd.). qPCR was performed in triplicate with SYBRII qPCR Master Mix (Takara Biotechnology Co., Ltd.) according to the manufacturer's protocol using GAPDH as a control.
RT was performed at $37^{\circ} \mathrm{C}$ for $15 \mathrm{~min}$ and $85^{\circ} \mathrm{C}$ for $5 \mathrm{~min}$. Thermocycling conditions were as follows: Initial denaturation at $95^{\circ} \mathrm{C}$ for $30 \mathrm{sec}$, followed by 40 cycles of $95^{\circ} \mathrm{C}$ for $5 \mathrm{sec}$ and $60^{\circ} \mathrm{C}$ for $31 \mathrm{sec}$. The relative mRNA levels of the target genes were calculated with the $2^{-\Delta \Delta \mathrm{Cq}}$ method (22). The primer sequences used in qPCR are listed in Table I.

Western blotting. Western blotting was performed as previously described (18). Briefly, total protein was extracted from $\operatorname{RBE}\left(5 \times 10^{6}\right)$ cells, CCA tissues and paracancerous tissues with cold RIPA buffer containing protease and phosphatase inhibitors (all Beijing Solarbio Science and Technology Co., Ltd.), followed by quantification of protein concentration using the BCA assay. Protein samples (30 $\mu \mathrm{g} /$ lane) were heated to $100^{\circ} \mathrm{C}$ for $10 \mathrm{~min}$ in SDS-PAGE loading buffer (Thermo Fisher Scientific, Inc.) and separated on 10\% SDS-PAGE. The separated proteins were transferred at $200 \mathrm{~mA}$ to a PVDF membrane (MilliporeSigma) for $2 \mathrm{~h}$ at $4^{\circ} \mathrm{C}$. After blocking the membrane with TBS containing $5 \%$ skimmed milk for $1.5 \mathrm{~h}$ at room temperature, the membranes were incubated with primary antibodies at $4^{\circ} \mathrm{C}$ overnight. The primary antibodies used were as follows: $\beta$-actin (rabbit monoclonal; 1:2,000; Abcam), Bax (rabbit monoclonal; 1:500; Abcam), Bcl2 (rabbit monoclonal antibody, dilution: 1:500; Abcam), cleaved caspase-3 (rabbit monoclonal antibody dilution: 1:500; Abcam), cleaved caspase-8 (rabbit polyclonal antibody, dilution: 1:500; Abcam), cleaved caspase-9 (rabbit polyclonal; 1:500; Abcam), MMP2 (rabbit polyclonal antibody, dilution: 1:500; Abcam), MMP9 (rabbit polyclonal antibody, dilution: 1:500; Abcam), ICAM-1 (rabbit polyclonal antibody, dilution: 1:500; Abcam); VEGFA (rabbit polyclonal; 1:500; Abcam); E-cadherin (rabbit polyclonal; 1:500; Abcam); N-cadherin (rabbit polyclonal antibody, dilution: 1:500; Abcam); TWIST (mouse monoclonal antibody, dilution: 1:500, Santa Cruz Biotechnology, Inc.); Vimentin (mouse monoclonal antibody, dilution: 1:500; Santa Cruz Biotechnology, Inc.); CSRC (rabbit monoclonal; 1:500; Abcam); p-CSRC (rabbit monoclonal antibody, dilution: 1:500; Abcam); PI3K, p-PI3K, AKT, p-AKT (rabbit monoclonal antibody, dilution: 1:500; Cell Signaling Technology, Inc.). Appropriate horseradish peroxidase-conjugated secondary antibody (goat anti-rabbit IgG, dilution: 1:5,000 and goat anti-mouse IgG, dilution: $1: 3,000$ ) at $25^{\circ} \mathrm{C}$ for $1 \mathrm{~h}$. The bands were detected using SuperECL Plus detection reagents (LI-COR Biosciences) and quantified with a Bio-Rad ChemiDoc imaging system (Bio-Rad Laboratories, Inc.) using $\beta$-actin as an internal control.

Cell transfection. RBE cells (30-40\% confluence) were transfected with Lipofectamine ${ }^{\circledR}$ RNAiMAX Reagent (Thermo Fisher Scientific, Inc.) and $20 \mathrm{nM}$ small interfering RNA (siRNA) (Guangzhou RiboBio Co., Ltd.) according to the manufacturer's instructions for $4 \mathrm{~h}$ at $37^{\circ} \mathrm{C}$. The sequences of all specific and control siRNAs are as follows: siRNA-1 CCUCUACAAAGAGGUGGACAGAGAATT; siRNA-2 CCA UGGUGAGAAAUUGACGACUUCATT; siRNA-3 CAC CACUGCCAAGCUCACUAUUGAATT and control, CCU CUUACCUCAGUUACAAUUUAUATT. Three separate CEACAM6-specific siRNA sequences were used, and an unrelated control siRNA sequence was transfected using the same protocol, which had no effect on CEACAM6 expression. 
Table I. Primer sequences for quantitative PCR.

\begin{tabular}{lll}
\hline Gene & \multicolumn{1}{c}{ Forward (5'-3') } & \multicolumn{1}{c}{ Reverse $\left(3^{\prime}-5^{\prime}\right)$} \\
\hline MMP-2 & CCAACTACAACTTCTTCCCTCG & TCACATCGCTCCAGACTTG \\
MMP-9 & ACGCAGACATCGTCATCCA & AGGGACCACAACTCGTCATC \\
ICAM-1 & GTCATCATCACTGTGGTAGCAG & GGCTTGTGTGTTCGGTTTC \\
E-cadherin & GTGGTCAAAGAGCCCTTACTG & CGTTACGAGTCACTTCAGGC \\
N-cadherin & TCATTGCCATCCTGCTCTG & CATCCATACCACAAACATCAGC \\
Vimentin & AAATGGCTCGTCACCTTCG & AGAAATCCTGCTCTCCTCGC \\
TWIST1 & GGAGTCCGCAGTCTTACGA & CTTGAGGGTCTGAATCTTGCT \\
Bcl-2 & TGTGTGGAGAGCGTCAACC & TGGATCCAGGTGTGCAGGT \\
Bax & TTTCTGACGGCAACTTCAAC & AGTCCAATGTCCAGCCCAT \\
Caspase 3 & ACTGGACTGTGGCATTGAGAC & TTGTCGGCATACTGTTTCAGC \\
Caspase 8 & TGTTGGAGGAAAGCAATCTG & CTGCCTGGTGTCTGAAGTTC \\
Caspase 9 & GCAGATTTGGCTTACATCCTG & ACGGCAGAAGTTCACATTGT \\
VEGFA & CACTATTGAATCCACGCCG & TTGCCATCCACTCTTTCG \\
c-SRC & CTTGCCTTGCTGCTCTACCT & TGATGATTCTGCCCTCCTCCT \\
PI3K & GAGGAGCCCATTTACATCG & CTTGAGAAAGTCCAGCAAACTC \\
AKT & GCCTGCTCTGTAGTGGTAGATG & GGAGGTGTGTTGGTAATGTAGC \\
\hline
\end{tabular}

ICAM-1, intercellular cell adhesion molecule-1; TWIST1, Twist-related protein 1; c-SRC, cellular SRC; CEACAM6, carcinoembryonic antigen-related cell adhesion molecule 6 .

Non-transfected cells were also used as a negative control; these were treated with serum-free DMEM for $4 \mathrm{~h}$ at $37^{\circ} \mathrm{C}$. Knockdown of CEACAM6 expression was confirmed at $48 \mathrm{~h}$ by western blotting.

Cell viability determination. The viability of RBE cells was determined using a Cell Counting Kit (CCK)-8 assay (Abcam) according to the manufacturer's instructions. Briefly, transfected cells $\left(2 \times 10^{3}\right)$ in 96 -well plates were incubated for $24 \mathrm{~h}$ at $37^{\circ} \mathrm{C}$ and $5 \% \mathrm{CO}_{2}$. CCK-8 reagent $(10 \mu \mathrm{l})$ was added for $1 \mathrm{~h}$ at $37^{\circ} \mathrm{C}$ and optical density was measured at $460 \mathrm{~nm}$.

Cell apoptosis analysis. RBE cells (80-90\% confluence) transfected with siRNAs for $48 \mathrm{~h}$ were washed twice with PBS (both $30 \mathrm{sec}$ at room temperature) and then resuspended in staining buffer containing $0.025 \mathrm{mg} / \mathrm{ml}$ Annexin V-FITC and $1 \mathrm{mg} / \mathrm{ml}$ propidium iodide (Shanghai Yisheng Biotechnology Co., Ltd.). Double staining was performed for $10 \mathrm{~min}$ at room temperature in the dark, and the number of apoptotic cells was then determined by flow cytometry using a BD FACSCanto ${ }^{\mathrm{TM}}$ II flow cytometer (BD Biosciences). The software used for data analysis is FlowJo (7.6.1; FlowJo, LLC.).

In vitro invasion assay. Cell invasion assays were performed using Transwell inserts (Corning, Inc.) coated with Matrigel $\left(37^{\circ} \mathrm{C}\right.$ for $\left.1 \mathrm{~h}\right)$ were performed as previously described (23). Briefly, RBE cells $\left(1 \times 10^{5}\right)$ in $0.2 \mathrm{ml}$ serum-free DMEM were placed in the upper chamber, and the lower chamber was loaded with $0.5 \mathrm{ml}$ medium containing $15 \%$ FBS. Cells that migrated to the lower surface of the filters were stained with $0.005 \%$ crystal violet solution for $40 \mathrm{~min}$ at room temperature, and cells in five fields of view were counted after $24 \mathrm{~h}$ of incubation at $37^{\circ} \mathrm{C}$ and $5 \% \mathrm{CO}_{2}$ using light microscope. Three wells were examined for each cell type and condition, and the experiments were conducted in triplicate.

Wound healing assay. RBE cells $\left(6 \times 10^{5}\right)$ were seeded in 6-well plates. When cells reached $90 \%$ confluency, a $100-\mu 1$ pipette tip was used to scratch the serum-starved cell monolayer (time $0 \mathrm{~h}$ ). Images of migrating cells during the closure of the wounded region were captured at 0 and $24 \mathrm{~h}$ using light microscope (magnification, x10).

Statistical analysis. All statistical analyses were conducted using SPSS version 19.0 (IBM, Corp.). Data are presented as the mean \pm standard error of the mean of three independent repeats. Comparisons of the means of different groups were performed by using two-way mixed ANOVA or one-way ANOVA. Pairwise comparisons with homogeneity of variance were performed using the post hoc Tukey's honestly significant difference and Student-Newman-Keuls tests. $\mathrm{P}<0.05$ was considered to indicate a statistically significant difference.

\section{Results}

CEACAM6 is overexpressed in CCA tissues, and its expression level is negatively associated with tumor differentiation degree. As shown in Fig. 1, CEACAM6 was overexpressed in highly differentiated and less differentiated CCA tissues compared with its expression levels in highly differentiated and less differentiated paracancerous tissues $(\mathrm{P}<0.05)$. Furthermore, lesser differentiation degrees of CCA tissues resulted in higher CEACAM6 expression levels $(\mathrm{P}<0.05)$. 


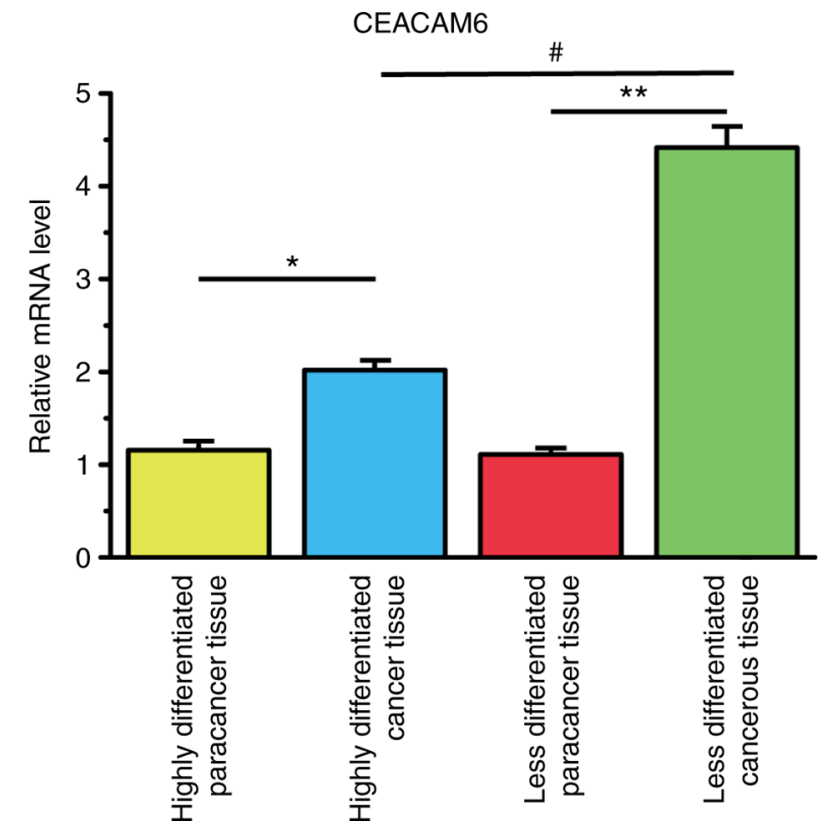

Figure 1. CEACAM6 expression in CCA and paracancerous tissues. Data are shown as the mean \pm standard error of the mean $(n=8)$, and were analyzed with two-way mixed ANOVA followed by Tukey's post hoc test. ${ }^{*} \mathrm{P}<0.05$ highly differentiated paracancerous tissues vs. highly differentiated CCA tissues; ${ }^{* *} \mathrm{P}<0.01$ less differentiated paracancerous tissues vs. less differentiated CCA tissues; ${ }^{\text {}} \mathrm{P}<0.05$ highly differentiated CCA tissues vs. less differentiated CCA tissues. CEACAM6, carcinoembryonic antigen-related cell adhesion molecule 6; CCA, cholangiocarcinoma.

Silencing CEACAM6 inhibits cell and promotes cell apoptosis in RBE cells. After determining that CEACAM6 was highly expressed in CCA tissues, the role of CEACAM6 in the invasion and migration of RBE cells was next verified. siRNA transfection was used to silence the expression of CEACAM6. The results of Fig. 2A-D show that CEACAM6-siRNA-2 exhibited the best knockdown efficiency after $48 \mathrm{~h}$. Thus, CEACAM6-siRNA-2 was utilized in subsequent experiments. As shown in Fig. 2E, CEACAM6-siRNA-2 significantly decreased RBE cell viability by $\sim 50$ compared with that of the control group $(\mathrm{P}<0.05)$. In addition, Fig. $2 \mathrm{~F}$ shows that the percentage of apoptotic cells increased after transfection of CEACAM6-siRNA-2 (34.4\%) compared with that of the scramble (scr)-siRNA group $(13.8 \% ; \mathrm{P}<0.05)$.

CEACAM6 knockdown inhibits the invasion and migration of RBE cells. As shown in Fig. 3A and C, the invasion ability of RBE cells was decreased after transfection with CEACAM6-siRNA-2. Similar results were obtained regarding the migration ability of $\mathrm{RBE}$ cells, which was also declined upon CEACA-siRNA-2 transfection, as shown in Fig. 3B and $\mathrm{D}(\mathrm{P}<0.05)$.

CEACAM6 silencing increases anti-apoptotic protein expression and decreases pro-apoptotic protein expression. Since the percentage of apoptotic cells increased after transfection with CEACAM6-siRNA-2, the present study next investigated whether the mRNA and protein expression levels of apoptosis-related proteins were consistent with the aforementioned findings. The results showed that the mRNA and protein levels of anti-apoptotic Bcl-2 decreased, while those of pro-apoptotic Bax increased after transfection with CEACAM6-siRNA-2.

The caspase family is composed of a class of proteolytic enzymes that mediate apoptosis and are activated during cell apoptosis. Caspases-3, -8 and -9 are major members of the caspase family. The results showed that the protein of cleaved caspases-3, -8 and -9 and mRNA levels of caspases-3, -8 and -9 were significantly upregulated after transfection with CEACAM6-siRNA-2 (Fig. 4A-G).

CEACAM6 silencing decreases EMT marker expression in $R B E$ cells. There are four types of EMT markers (epithelial and interstitial cell, transcription factor and cytoskeleton); of them, the epithelial cell marker E-cadherin and the interstitial cell marker $\mathrm{N}$-cadherin are the most studied. In the present study, E-cadherin expression was significantly increased, while $\mathrm{N}$-cadherin expression was significantly decreased after CEACAM6-siRNA-2 transfection.

MMPs are a group of zinc ion $\left(\mathrm{Zn}^{2+}\right)$-dependent endopeptidases that degrade extracellular matrix (ECM) and then induce pathological processes, such as tumor cell invasion and migration. MMP-2 and MMP-9 are important MMPs, and the protein and mRNA expression levels of these endopeptidases were reduced after siRNA transfection.

The TWIST transcription factor enhances EMT by inhibiting E-cadherin expression and enhancing tumor cell migration and invasion. The protein and mRNA expression of TWIST decreased after transfection with CEACAM6-siRNA-2.

Vimentin is an intermediate filament protein in mesenchymal cells that regulates protein-protein interactions, such as those involving cytoskeletal proteins and cell adhesion molecules, which may participate in cell invasion, migration and signal transduction. After transfection with CEACAM6-siRNA-2, the expression level of this tumor-promoting molecule decreased.

Furthermore, tumor angiogenesis is associated with VEGFA overexpression in human iCCA and pCCA (24). In the present study, the protein and mRNA levels of VEGFA and tumorigenesis-promoting ICAM-1 decreased when CEACAM6-siRNA-2 was transfected into RBE cells. The aforementioned results are shown in Fig. 4H-R.

CEACAM6 silencing decreases the expression of members of the SRC/PI3K/AKT signal transduction pathway in $R B E$ cells. The present study demonstrated that there was no significant difference in the relative mRNA levels of molecules involved in the SRC/PI3K/AKT signal transduction pathway when CEACAM6 was knocked down (Fig. 5A). However, when CEACAM6-siRNA-2 was added to the cells, the levels of phosphorylated molecules involved in the SRC/PI3K/AKT signal transduction pathway was reduced (Fig. 5B-E).

\section{Discussion}

CCA is the second most common type of primary liver cancer in human patients after HCC (5). The incidence and mortality rates of iCCA are increasing worldwide. Despite advances in the detection and treatment of metastatic CCA, mortality from 

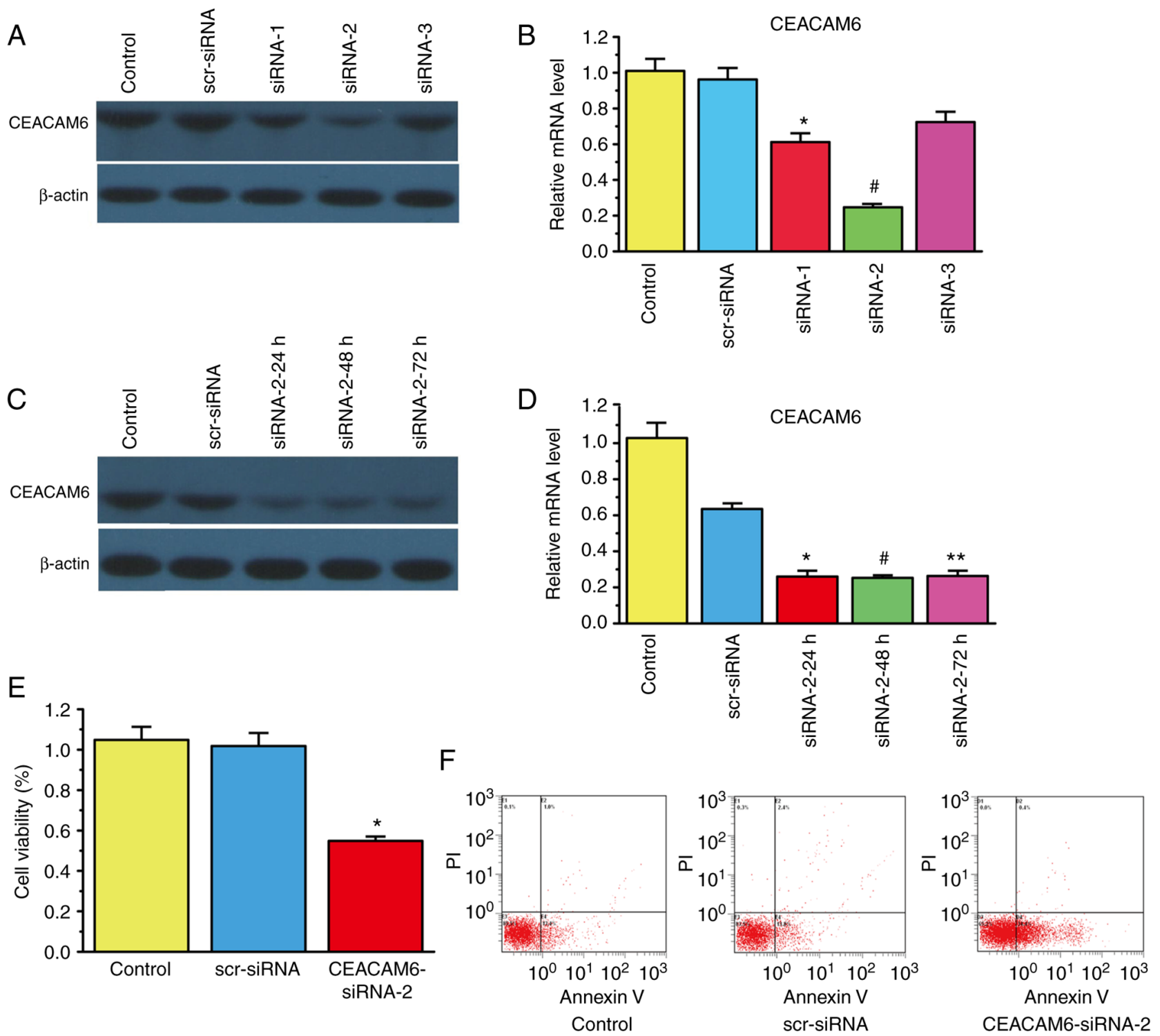

Figure 2. Effects of CEACAM6 silencing on RBE cell viability, apoptosis, invasion and migration in vitro. (A-D) Western blot and reverse transcription-quantitative PCR analyses were used to select siRNA and transfection time for CEACAM6 silencing. Data are shown as the mean \pm standard error of the mean $(\mathrm{n}=8)$. Data were analyzed with one-way ANOVA followed by Tukey's (4 groups) or Student-Newman-Keuls (3 groups) post hoc tests. For part B: "P<0.05 scr-siRNA vs. siRNA-1; ${ }^{\#} \mathrm{P}<0.05$ scr-siRNA vs. siRNA-2. For part $\mathrm{D}$ : " $\mathrm{P}<0.05$ scr-siRNA vs. siRNA-2-24 h; ${ }^{*} \mathrm{P}<0.05$ scr-siRNA vs. siRNA-2-48 h; ${ }^{* * *} \mathrm{P}<0.05$ scr-siRNA vs. siRNA-2-72 h. (E) Effects on cell viability of silencing CEACAM6 with CEACAM6-siRNA-2 after $48 \mathrm{~h}$ of transfection. "P<0.05 scr-siRNA vs. CEACAM6-siRNA. (F) Effects of silencing CEACAM6 by transfecting CEACAM6-siRNA-2 for $48 \mathrm{~h}$ on cell apoptosis. CEACAM6, carcinoembryonic antigen-related cell adhesion molecule 6; siRNA, small interfering RNA; scr, scramble.

this disease remains high due to its limited early-stage cytological and pathological diagnoses as well as limited effective treatments (1). Therefore, it is important to explore treatment methods based on specific markers and targets.

CEACAM6 is a member of the immunoglobulin superfamily, which is overexpressed in several human cancer types, including colorectal, pancreatic, lung and breast cancer $(20,25,26)$. A previous report has revealed that CEACAM6 expression is associated with adverse pathological features and prognosis in pancreatic cancer (14). In agreement with those findings, the present study demonstrated that CEACAM6 was highly expressed in CCA tissues and was negatively associated with the differentiation degree of CCA, suggesting that CEACAM6 may be involved in the development and progression of CCA. The present study demonstrated that CEACAM6 was highly expressed in CCA tissue and was negatively associated with degree of differentiation.

CEACAM6 is oncogenic, as it inhibits cell differentiation, causes loss of cell polarity, and promotes cell adhesion, invasion and metastasis (27). These oncogenic properties are inhibited by an anti-CEACAM6 antibody in breast, pancreatic and colorectal cancer (28). Similarly, the present in vitro results revealed that the invasion and migration of RBE cells decreased after CEACAM6-siRNA-2 transfection.

Apoptosis, or programmed cell death, is an essential physiological process that plays a critical role in development and tissue homeostasis (29). The caspase cascade plays vital roles in the induction, transduction and amplification of intracellular 
A
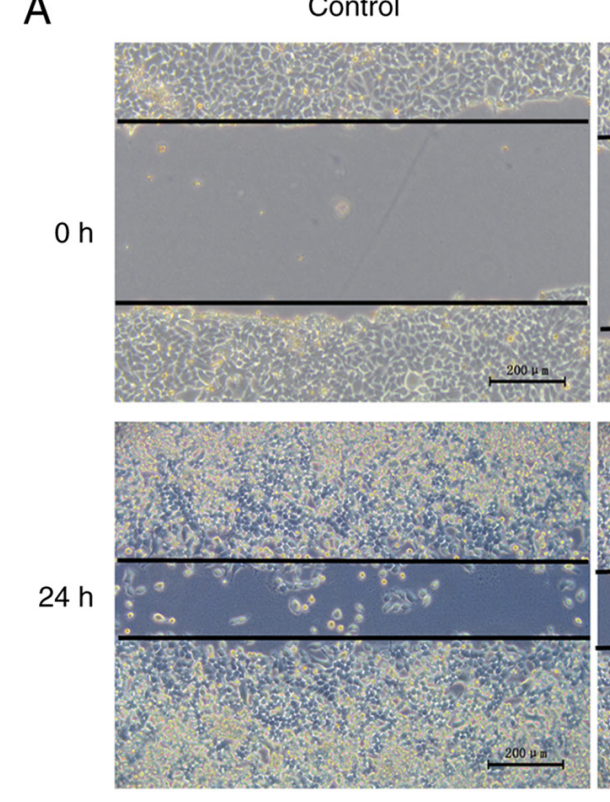

B

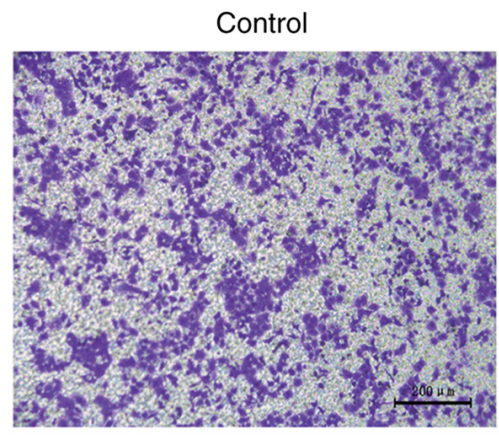

C

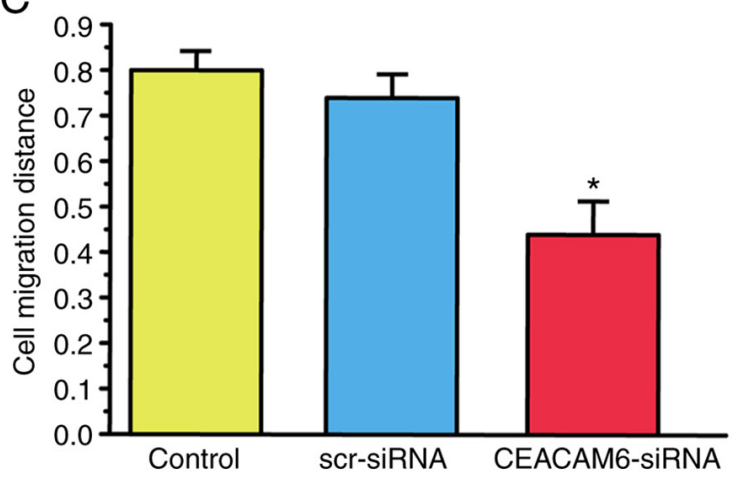

scr-siRNA
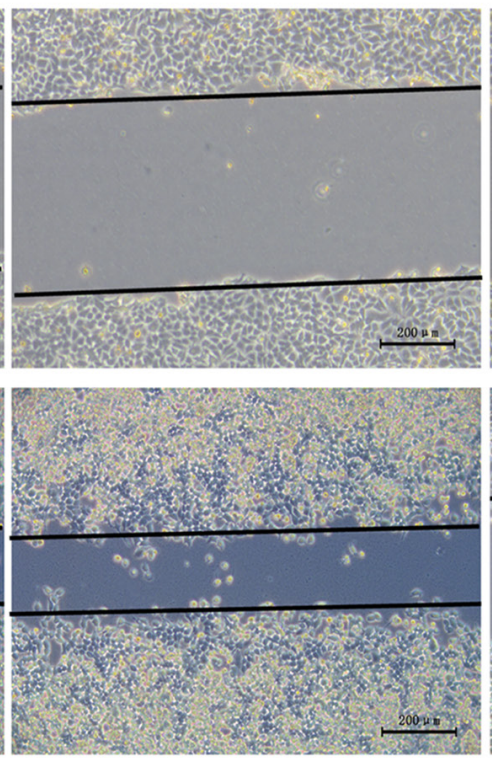

scr-siRNA

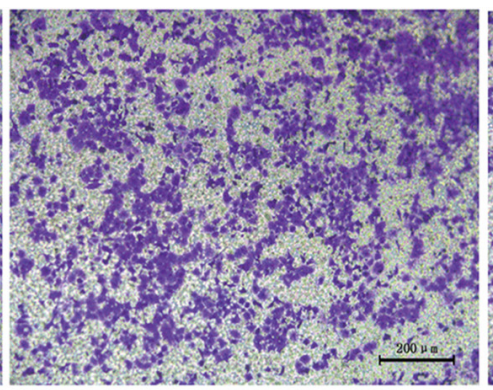

CEACAM6-SIRNA
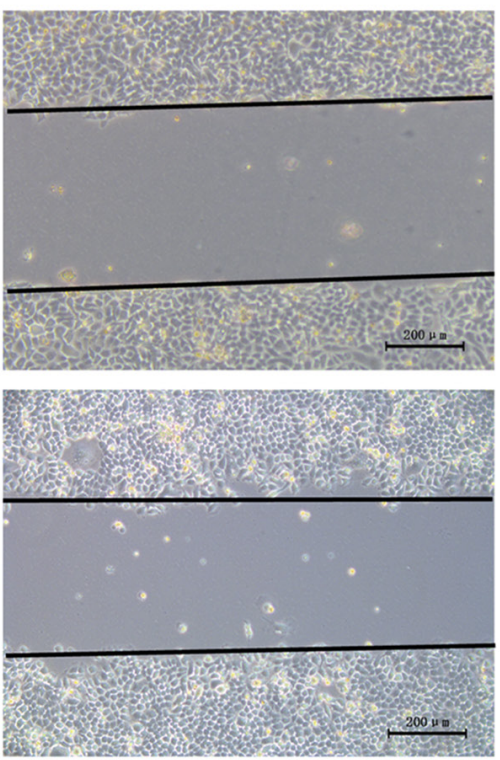

CEACAM6-SIRNA

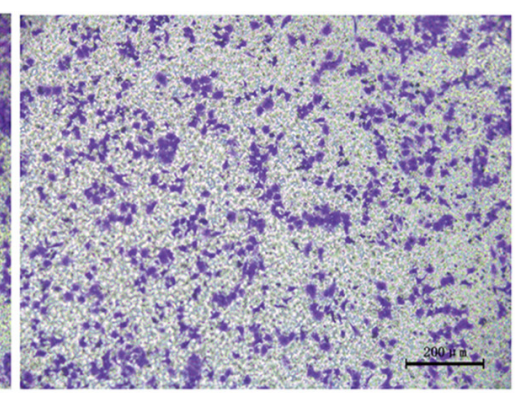

D

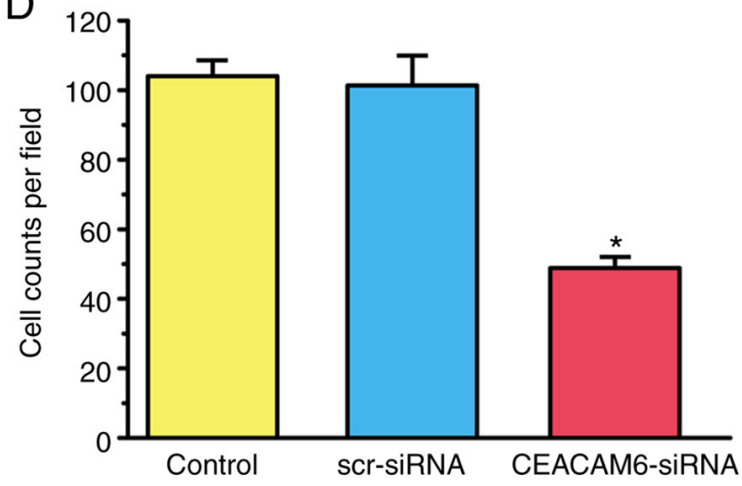

Figure 3. Effects CEACAM6 silencing on the invasion and migration of RBE cells in vitro. (A and C) Effects of silencing CEACAM6 via transfection of CEACAM6-siRNA-2 for $48 \mathrm{~h}$ on the wound healing and invasion abilities of RBE cells (magnification, x100; scale bar, $200 \mu \mathrm{m}$ ). (B and D) Effects of silencing CEACAM6 due to transfection of CEACAM6-siRNA-2 for $48 \mathrm{~h}$ on the invasion of RBE cells (magnification, $\mathrm{x} 100$; scale bar, $200 \mu \mathrm{m}$ ). Data are shown as the mean \pm standard error of the mean $(\mathrm{n}=8)$, and were analyzed with one-way ANOVA followed by Student-Newman-Keuls post hoc test, "P $<0.05$, scr-siRNA vs. CEACAM6-siRNA. CEACAM6, carcinoembryonic antigen-related cell adhesion molecule 6; siRNA, small interfering RNA; scr, scramble.

apoptotic signals. The present results showed that the protein and mRNA expression levels of cleaved caspases-3, -8 and -9 increased after transfection with CEACAM6-siRNA-2.

EMT has gained increased attention in the past years regarding metastatic dissemination (9). The present study demonstrated that CEACAM6 was an important regulator of EMT biomarkers in CCA. However, there may be other mechanisms involved in CCA progression, such as the role played by circulating tumor cells or that of exosomes released by different tumor cell types $(30,31)$, which suggests the need for further studies. Furthermore, a previous study has shown that the function of CEACAM6 is dependent on the c-SRC signaling pathway in pancreatic cancer (32).

In conclusion, the present study has demonstrated that CEACAM6 is significantly overexpressed in CCA tissues, which indicates that CEACAM6 could be utilized as a potential tumor EMT biomarker. Understanding the clinical significance of CEACAM6 expression and its oncogenic mechanism may eventually lead to the identification of a novel therapeutic target for human CCA treatment. 
A
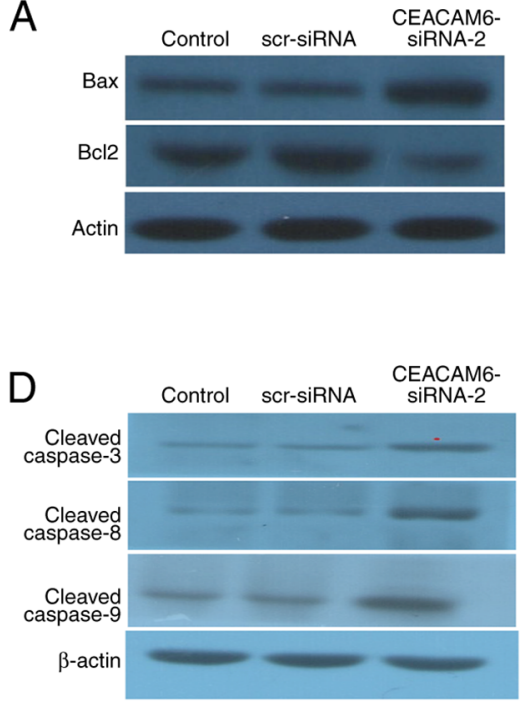

$\mathrm{H}$

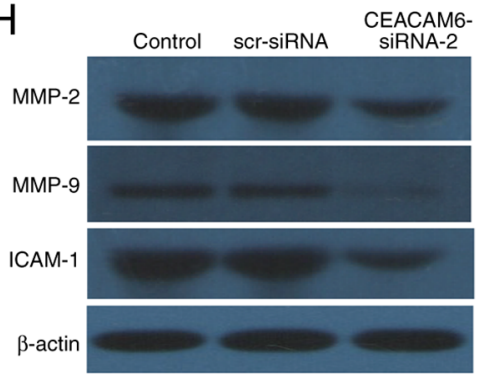

$\mathrm{K}$ CEACAMG-

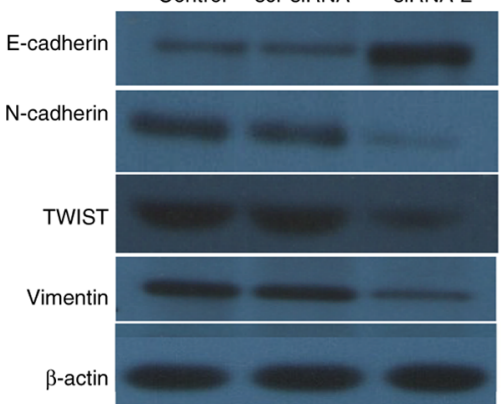

$\mathrm{P}$

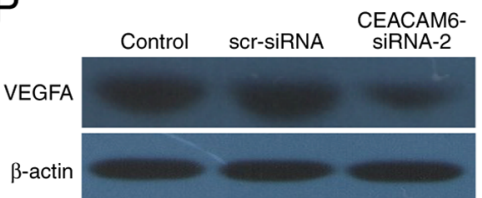

B

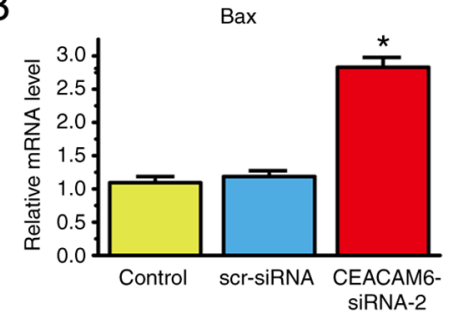

E

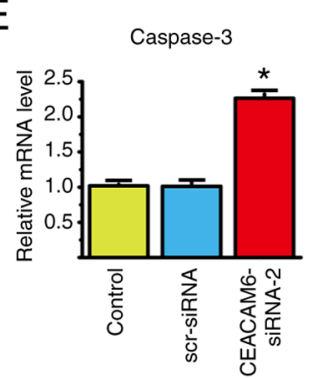

$\mathrm{F}$

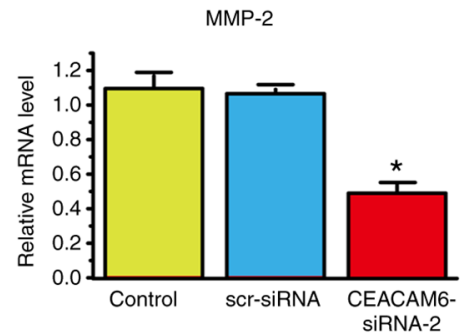

$\mathrm{L}$

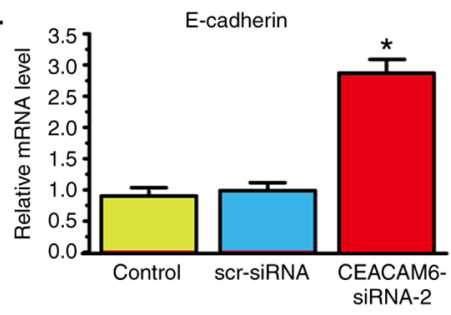

$\mathrm{N}$

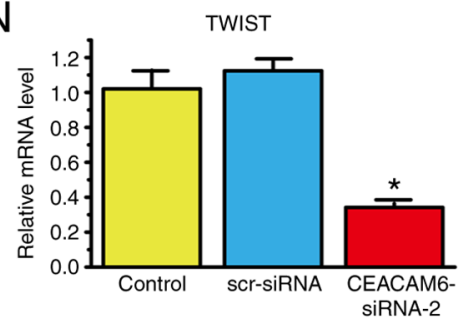

Q

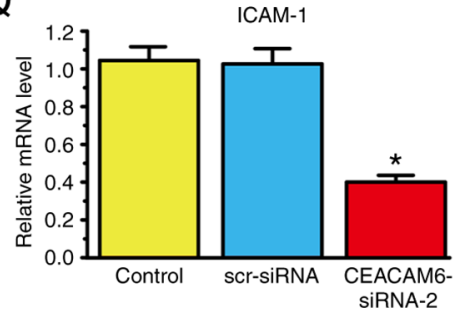

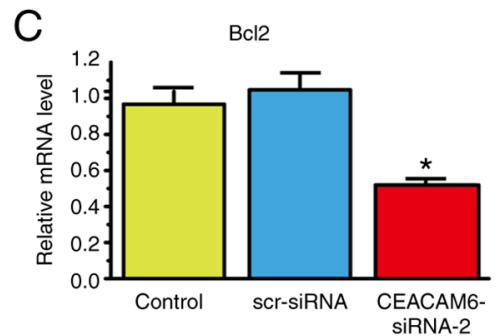

G

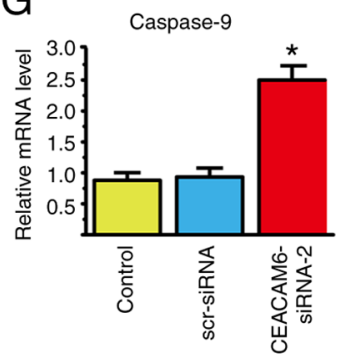

J

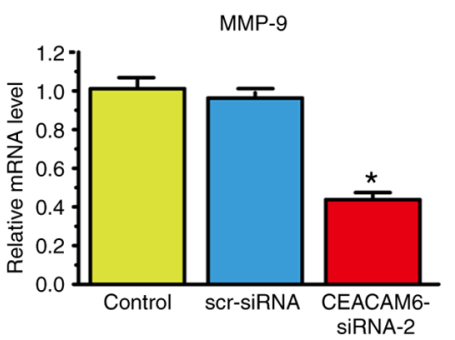

M

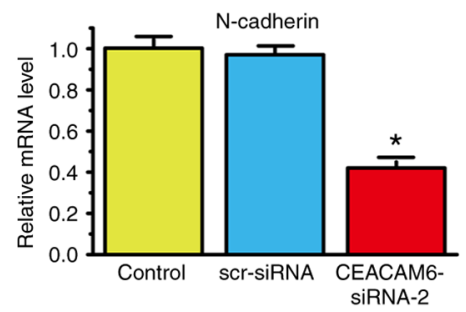

$\mathrm{O}$

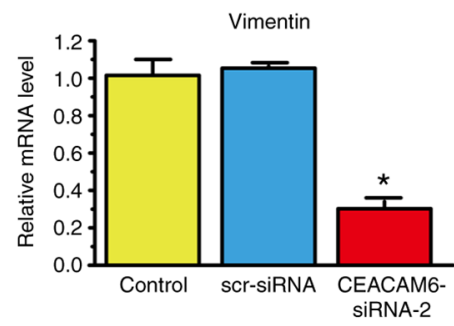

$\mathrm{R}$

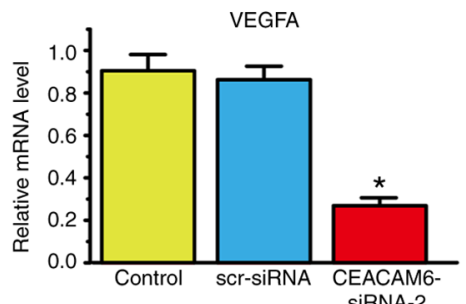

Figure 4. Effects of silencing CEACAM6 with CEACAM6-siRNA-2 after $48 \mathrm{~h}$ of transfection on the expression of apoptosis-related molecules and epithelial-mesenchymal transition markers. (A-G) Western blot and reverse transcription-quantitative PCR analyses were employed to detect the effects of silencing CEACAM6 on the expression of the pro-apoptotic protein Bax, cleaved caspases-3, -8 and -9 , and the anti-apoptotic protein Bcl-2. (H-R) Western blot and reverse transcription-quantitative PCR analyses were used to detect the effects of silencing CEACAM6 on the expression of the extracellular matrix-related proteins MMP-2 and MMP-9, the epithelial cell marker E-cadherin, the interstitial cell marker N-cadherin, the intermediate filament protein vimentin, the transcription factor TWIST, the tumor nutrient vascular formation-related molecule VEGFA, and the tumorigenesis-promoting factor ICAM-1. Data are shown as the mean \pm standard error of the mean $(n=8)$, and were analyzed with one-way ANOVA followed by Student-Newman-Keuls post hoc test. ${ }^{*} \mathrm{P}<0.05$ scr-siRNA vs. CEACAM6-siRNA. CEACAM6, carcinoembryonic antigen-related cell adhesion molecule 6; siRNA, small interfering RNA; scr, scramble; ICAM-1, intercellular cell adhesion molecule-1; TWIST, Twist-related protein. 
A
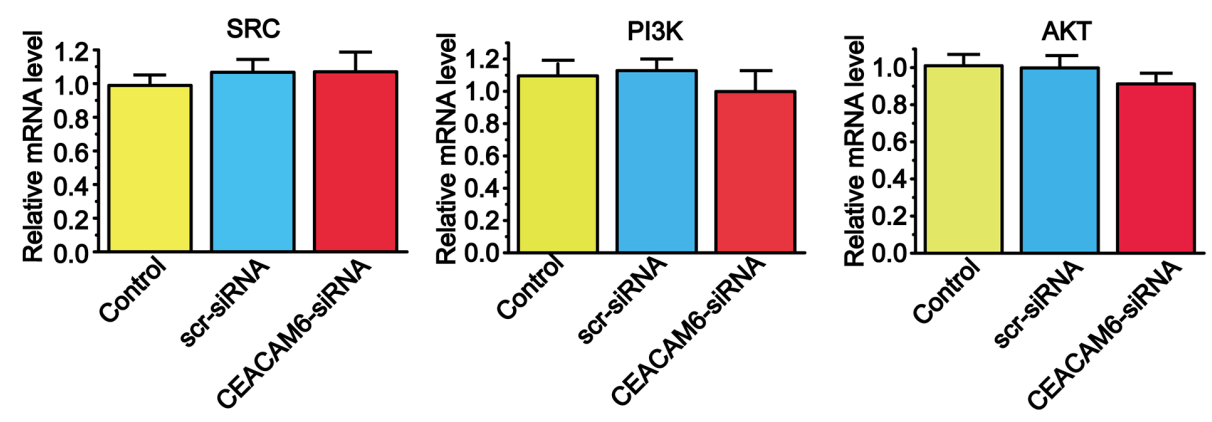

B

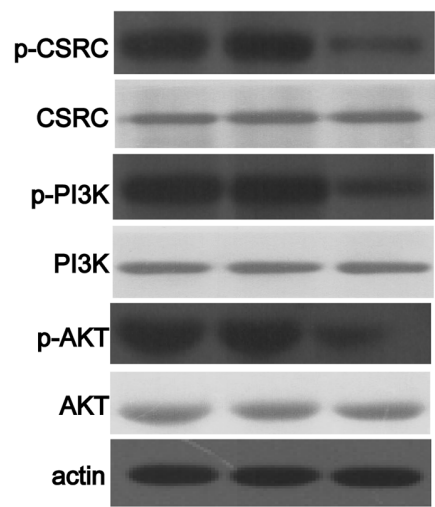

C

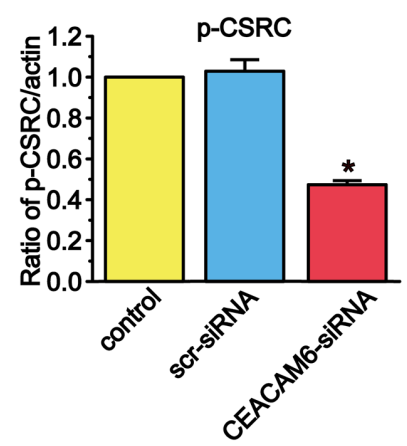

$\mathrm{E}$
D

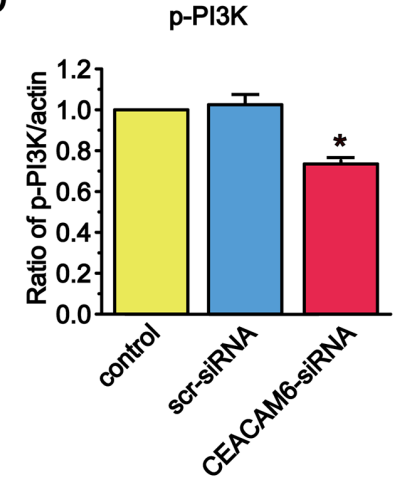

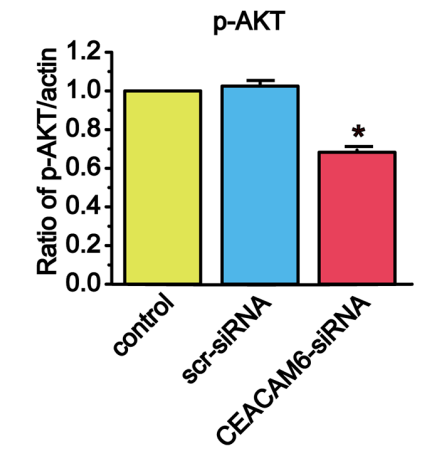

Figure 5. Effects of silencing CEACAM6 with CEACAM6-siRNA-2 after $48 \mathrm{~h}$ of transfection on the expression of members of the SRC/PI3K/AKT signal transduction pathway. (A) Reverse transcription-quantitative PCR analyses were performed to detect the effects of CEACAM6 silencing on the relative mRNA levels of SCR, PI3K and AKT. (B-E) Western blot analyses were conducted to detect the effects of CEACAM6 silencing on the levels of proteins associated with signal transduction (p-c-SRC, p-PI3K and p-AKT). Data are shown as the mean \pm standard error of the mean (n=8) and were analyzed with one-way ANOVA followed by Student-Newman-Keuls post hoc test. "P $<0.05$ scr-siRNA vs. CEACAM6-siRNA. CEACAM6, carcinoembryonic antigen-related cell adhesion molecule 6; siRNA, small interfering RNA; c-SRC, cellular; p-, phosphorylated; scr, scramble.

\section{Acknowledgements}

Not applicable.

\section{Funding}

The present study was supported by the Hebei Science and Technology Plan 2015 (grant no. 152377768D).

\section{Availability of data and materials}

All data generated or analyzed during this study are included in this published article.

\section{Authors' contributions}

CL performed the experiments and acquired data. MW analyzed and processed data, and wrote and revised the manuscript. HL,
$\mathrm{BL}, \mathrm{XY}$ and $\mathrm{WZ}$ analyzed and interpretated data. HL, BL, $\mathrm{XY}$ and WZ confirm the authenticity of all the raw data. WW designed the experiments and gave final approval of the version to be published. All authors read and approved the final version of the manuscript.

\section{Ethics approval and consent to participate}

Ethics approval was from The Second Hospital of Hebei Medical University. Written informed consent was obtained from all patients.

\section{Patient consent for publication}

Not applicable.

\section{Competing interests}

The authors declare that they have no competing interests. 


\section{References}

1. Bridgewater J, Galle PR, Khan SA, Llovet JM, Park JW, Patel T, Pawlik TM and Gores GJ: Guidelines for the diagnosis and management of intrahepatic cholangiocarcinoma. J Hepatol 60 1268-1289, 2014

2. Saha SK, Zhu AX, Fuchs CS and Brooks GA: Forty-year trends in cholangiocarcinoma incidence in the U.S.: Intrahepatic disease on the rise. Oncologist 21: 594-599, 2016.

3. Kayhanian H, Smyth EC and Braconi C: Emerging molecular targets and therapy for cholangiocarcinoma. World J Gastrointest Oncol 9: 268-280, 2017.

4. Khan SA, Taylor-Robinson SD, Toledano MB, Beck A, Elliott P and Thomas HC: Changing international trends in mortality rates for liver, biliary and pancreatic tumours. J Hepatol 37: 806-813, 2002.

5. Rizvi S, Khan SA, Hallemeier CL, Kelley RK and Gores GJ: Cholangiocarcinoma-evolving concepts and therapeutic strategies. Nat Rev Clin Oncol 15: 95-111, 2018.

6. Battaglia S, Benzoubir N, Nobilet S, Charneau P, Samuel D, Zignego AL, Atfi A, Brechot $\mathrm{C}$ and Bourgeade MF: Liver cancer-derived hepatitis $\mathrm{C}$ virus core proteins shift TGF-beta responses from tumor suppression to epithelial-mesenchymal transition. PLoS One 4: e4355, 2009.

7. Vaquero J, Guedj N, Claperon A, Nguyen Ho-Bouldoires TH, Paradis V and Fouassier L: Epithelial-mesenchymal transition in cholangiocarcinoma: From clinical evidence to regulatory networks. J Hepatol 66: 424-441, 2017.

8. Puisieux A, Brabletz T and Caramel J: Oncogenic roles of EMT-inducing transcription factors. Nat Cell Biol 16: 488-494, 2014.

9. Zhu JY, Zhou F, Yu L and Zhang J: Epithelial-mesenchymal transition of small airway epithelium in patients receiving lung tumor surgery with normal lung function and chronic obstructive pulmonary disease. Zhonghua Yi Xue Za Zhi 99: 2681-2686, 2019 (In Chinese).

10. Baum B, Settleman J and Quinlan MP: Transitions between epithelial and mesenchymal states in development and disease. Semin Cell Dev Biol 19: 294-308, 2008.

11. Zeisberg M and Neilson EG: Biomarkers for epithelial-mesenchymal transitions. J Clin Invest 119: 1429-1437, 2009.

12. Rizeq B, Zakaria $\mathrm{Z}$ and Ouhtit $\mathrm{A}$ : Towards understanding the mechanisms of actions of carcinoembryonic antigen-related cell adhesion molecule 6 in cancer progression. Cancer Sci 109 33-42, 2018.

13. Yamanka T, Kuroki M, Matsuo Y and Matsuoka Y: Analysis of heterophilic cell adhesion mediated by CD66b and CD66c using their soluble recombinant proteins. Biochem Biophys Res Commun 219: 842-847, 1996

14. Ieta K, Tanaka F, Utsunomiya T, Kuwano $\mathrm{H}$ and Mori M: CEACAM6 gene expression in intrahepatic cholangiocarcinoma. Br J Cancer 95: 532-540, 2006.

15. Rose JB, Correa-Gallego C, Li Y, Nelson J, Alseidi A, Helton WS, Allen PJ, D'Angelica MI, DeMatteo RP, Fong Y, et al: The role of biliary carcinoembryonic antigen-related cellular adhesion molecule 6 (CEACAM6) as a biomarker in cholangiocarcinoma. PLoS One 11: e0150195, 2016.

16. Farina A, Dumonceau JM, Antinori P, Annessi-Ramseyer I, Frossard JL, Hochstrasser DF, Delhaye M and Lescuyer P: Bile carcinoembryonic cell adhesion molecule 6 (CEAM6) as a biomarker of malignant biliary stenoses. Biochim Biophys Acta 1844: 1018-1025, 2014.

17. Kuespert K, Pils S and Hauck CR: CEACAMs: Their role in physiology and pathophysiology. Curr Opin Cell Biol 18 : 565-571, 2006.
18. Ilantzis C, DeMarte L, Screaton RA and Stanners CP: Deregulated expression of the human tumor marker CEA and CEA family member CEACAM6 disrupts tissue architecture and blocks colonocyte differentiation. Neoplasia 4: 151-163, 2002.

19. Ordonez C, Screaton RA, Ilantzis C and Stanners CP: Human carcinoembryonic antigen functions as a general inhibitor of anoikis. Cancer Res 60:3419-3424, 2000.

20. Duxbury MS, Ito H, Zinner MJ, Ashley SW and Whang EE: CEACAM6 gene silencing impairs anoikis resistance and in vivo metastatic ability of pancreatic adenocarcinoma cells. Oncogene 23: 465-473, 2004.

21. Chen J, Li Q, An Y, Lv N, Xue X, Wei J, Jiang K, Wu J, Gao W, Qian Z, et al: CEACAM6 induces epithelial-mesenchymal transition and mediates invasion and metastasis in pancreatic cancer. Int J Oncol 43: 877-885, 2013.

22. Livak KJ and Schmittgen TD: Analysis of relative gene expression data using real-time quantitative PCR and the 2(-Delta Delta C(T)) method. Methods 25: 402-408, 2001.

23. Zhang Y, Zang M, Li J, Ji J, Zhang J, Liu X, Qu Y, Su L, Li C, $\mathrm{Yu}$ Y, et al: CEACAM6 promotes tumor migration, invasion, and metastasis in gastric cancer. Acta Biochim Biophys Sin (Shanghai) 46: 283-290, 2014.

24. Mancarella S, Serino G, Dituri F, Cigliano A, Ribback S, Wang J, Chen X, Calvisi DF and Giannelli G: Crenigacestat, a selective NOTCH1 inhibitor, reduces intrahepatic cholangiocarcinoma progression by blocking VEGFA/DLL4/MMP13 axis. Cell Death Differ 27: 2330-2343, 2020.

25. Kim KS, Kim JT, Lee SJ, Kang MA, Choe IS, Kang YH, Kim SY, Yeom YI, Lee YH, Kim JH, et al: Overexpression and clinical significance of carcinoembryonic antigen-related cell adhesion molecule 6 in colorectal cancer. Clin Chim Acta 415: 12-19, 2013.

26. Lewis-Wambi JS, Cunliffe HE, Kim HR, Willis AL and Jordan VC: Overexpression of CEACAM6 promotes migration and invasion of oestrogen-deprived breast cancer cells. Eur J Cancer 44: 1770-1779, 2008.

27. Kobayashi M, Miki Y, Ebina M, Abe K, Mori K, Narumi S, Suzuki T, Sato I, Maemondo M, Endo C, et al: Carcinoembryonic antigen-related cell adhesion molecules as surrogate markers for EGFR inhibitor sensitivity in human lung adenocarcinoma. $\mathrm{Br} \mathbf{J}$ Cancer 107: 1745-1753, 2012.

28. Zang M, Zhang B, Zhang Y, Li J, Su L, Zhu Z, Gu Q, Liu B and Yan M: CEACAM6 promotes gastric cancer invasion and metastasis by inducing epithelial-mesenchymal transition via PI3K/AKT signaling pathway. PLos One 9: e112908, 2014.

29. Fan TJ, Han LH, Cong RS and Liang J: Caspase family proteases and apoptosis. Acta Biochim Biophys Sin (Shanghai) 37: 719-727, 2005.

30. Makler A and Asghar W: Exosomal biomarkers for cancer diagnosis and patient monitoring. Expert Rev Mol Diagn 20: $387-400,2020$

31. Deng Z, Wu J, Xu S, Chen F, Zhang Z, Jin A and Wang J: Exosomes-microRNAs interacted with gastric cancer and its microenvironment: A mini literature review. Biomark Med 14: 141-150, 2020.

32. Duxbury MS, Ito H, Ashley SW and Whang EE: c-Src-dependent cross-talk between CEACAM6 and alphavbeta3 integrin enhances pancreatic adenocarcinoma cell adhesion to extracellular matrix components. Biochem Biophys Res Commun 317: 133-141, 2004.

This work is licensed under a Creative Commons Attribution-NonCommercial-NoDerivatives 4.0 International (CC BY-NC-ND 4.0) License. 